\title{
Novel Non-invasive Magnetic Resonance Imaging Methods in Cerebrovascular Disease
}

\author{
Manus J Donahue ${ }^{1}$ and Megan K Strother ${ }^{2}$ \\ 1. Assistant Professor, Departments of Radiology, Neurology, Psychiatry, and Physics and Astronomy; \\ 2. Associate Professor, Department of Radiology, School of Medicine, Vanderbilt University Nashville, Tennessee, us
}

\begin{abstract}
Neuroimaging is a critical component of patient care in multiple stages of cerebrovascular disease. Most imaging focuses on measurements of tissue or vascular structure, with comparatively less emphasis on function. Furthermore, imaging approaches that rely on exogenous contrast agents or ionising radiation are common and provide crucial information regarding treatment decisions; however, they are suboptimal for monitoring patients longitudinally or in response to therapy due to dose restrictions and related health concerns. We review the state of non-invasive magnetic resonance imaging (MRI) approaches that have demonstrated clinical potential in patients with cerebrovascular disease, yet have not been incorporated into routine radiological protocols at most hospitals. These approaches include blood oxygenation level-dependent (BOLD) for cerebrovascular reactivity, arterial spin labelling (ASL) for cerebral blood flow quantification, chemical exchange saturation transfer (CEST) for macromolecule and pH determination and arterial vessel wall imaging for plaque visualisation. The strengths and limitations of these approaches are presented, as well as a summary of their implementation in stroke.
\end{abstract}

\section{Keywords}

Stroke, BOLD, ASL, CEST, reactivity, neurovascular imaging

Disclosure: The authors have no conflicts of interest to declare.

Acknowledgements: This work was supported in part by the National Institutes of Health (NIH)/National Institute of Neurological Disorders and Stroke (NINDS) (5R01NS078828-02).

Received: 16 August 2013 Accepted: 30 October 2013 Citation: European Neurological Review, 2013;8(2):153-8 DOI:10.17925/ENR.2013.08.02.153

Correspondence: Manus J Donahue, Vanderbilt University Institute of Imaging Science, 1161 21st Ave N, MCN, AAA-3115, Nashville, TN 37232-2310, US.

E: mj.donahue@vanderbilt.edu

Stroke is the leading cause of adult disability and the second leading cause of death in developed countries. ${ }^{1}$ Despite progress in stroke treatment, 20-30\% of strokes result in death within one month and 70-80 \% result in significant long-term disability..$^{2-4}$ Improved preventative and acute management of cerebrovascular disease has reduced stroke-related mortality: ${ }_{i}^{5}$ however, many stroke survivors remain impaired with nearly $33 \%$ institutionalised after stroke. ${ }^{6-9}$

Neuroimaging is a critical component of patient care in all stages of cerebrovascular disease: from identification of stroke risk factors to stratifying acute and chronic stroke patients to the most effective revascularisation and rehabilitation treatments. Evaluation of management decisions and post-stroke therapy strategies would be accelerated with an improved understanding of the complex interplay between vascular, neurochemical and tissue-level haemodynamic impairment.

Characterisation of soft-tissue structure using fluid attenuated inversion recovery (FLAIR) and T1-weighted magnetic resonance imaging (MRI) is central to most standard MRI protocols and extensive work has documented the relevance of these techniques..$^{10-12}$ In more specialised protocols, diffusion weighted imaging (DWI) or diffusion tensor imaging (DTI) can be applied to characterise membrane integrity and fibre tract directionality, respectively, ${ }^{13}$ and neurochemical tissue signatures can be assessed using principles of magnetic resonance spectroscopy. ${ }^{14}$ These relatively well-known approaches have been implemented in patients with cerebrovascular disease for more than two decades. However, newer approaches are continuously being developed that hold potential for further expanding our understanding of the functional and structural sequelae of tissue changes during and following ischaemia.

Here, we review the state of novel, non-invasive MRI approaches capable of complementing the existing stroke imaging infrastructure for more comprehensive evaluation of cerebrovascular disease. As each method is in a different stage of development, with varying timelines for routine clinical implementation, the purpose of this article is to review these emerging methods in the context of their strengths and limitations for evaluating cerebrovascular disease and to provide references relevant for further evaluation of their existing impact on stroke imaging (see Table 1).

\section{Magnetic Resonance Imaging}

MRI has been routinely used clinically for more than 20 years, with a primary purpose for visualising structural soft-tissue contrast. The main signal source in MRI is water and the contrast derives from how the magnetic properties (Iongitudinal and transverse relaxation times: T1 and $\mathrm{T} 2$, respectively) of water protons in different environments adjust in the presence of a static magnetic field (BO; generally 1.5-3.0 Tesla) 
Table 1: Non-invasive Magnetic Resonance Imaging Approaches with Demonstrated Clinical Potential in Cerebrovascular Disease, Listed in Order of Decreasing Clinical Feasibility Testing

\begin{tabular}{|c|c|c|c|c|c|c|}
\hline Technique & $\begin{array}{l}\text { Primary Parameter(s) } \\
\text { Measured }\end{array}$ & $\begin{array}{l}\text { Other Parameters } \\
\text { Measured }\end{array}$ & Duration & $\begin{array}{l}\text { Spatial } \\
\text { Resolution }\end{array}$ & $\begin{array}{l}\text { Clinical Stroke } \\
\text { Reference }\end{array}$ & $\begin{array}{l}\text { Methodological } \\
\text { Reference }\end{array}$ \\
\hline BOLD Reactivity & CBF and CBV reactivity & $\mathrm{CMRO}_{2}$ & 5-12 minutes & 3-4 mm & $\begin{array}{l}\text { Mandell et al. }{ }^{90} \\
\text { Donahue et al. }{ }^{37}\end{array}$ & $\begin{array}{l}\text { Buxton et al. }{ }^{91} \\
\text { van Zijl et al. }{ }^{26}\end{array}$ \\
\hline ASL & CBF & Arterial arrival time & 3-8 minutes & $3-5 \mathrm{~mm}$ & $\begin{array}{l}\text { Tancredi et al. }{ }^{92} \\
\text { Chalela et al. } .^{93}\end{array}$ & $\begin{array}{l}\text { Detre et al. }{ }^{94} \\
\text { Okell et al. }{ }^{95}\end{array}$ \\
\hline AWI & Vessel wall thickness & Plaque & 6-11 minutes & $0.5-1 \mathrm{~mm}$ & $\begin{array}{l}\text { van der Kolk et al. }{ }^{96} \\
\text { Mandell et al. }{ }^{97}\end{array}$ & $\begin{array}{l}\text { van der Kolk et al. }{ }^{98} \\
\text { Li et al. } .^{87}\end{array}$ \\
\hline CEST & $\mathrm{pH}$ & $\begin{array}{l}\text { Macro-molecules with } \\
\text { labile protons }\end{array}$ & 3-7 minutes & $1-3 \mathrm{~mm}$ & $\begin{array}{l}\text { Sun et al. }{ }^{99} \\
\text { Tietze et al. }{ }^{100}\end{array}$ & $\begin{array}{l}\text { van Zijl et al. }{ }^{101} \\
\text { Ward et al. }{ }^{59}\end{array}$ \\
\hline
\end{tabular}

$A S L=$ arterial spin labelling; $A W I=$ arterial wall imaging; $B O L D=$ blood oxygenation level-dependent; $C B F=$ cerebral blood flow; $C B V=$ cerebral blood volume; $C E S T=$ chemical exchange saturation transfer; $\mathrm{CMRO}_{2}=$ cerebral metabolic rate of oxygen consumption.

\section{Figure 1: Blood Oxygenation Level-dependent Cerebrovascular Reactivity}

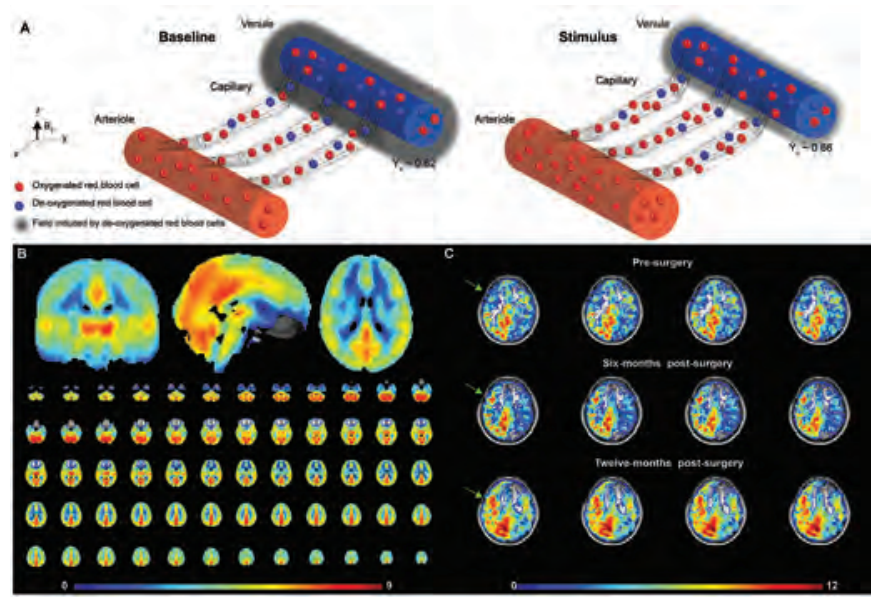

A: Physiology underlying the blood oxygenation level-dependent (BOLD) effect. During either neuronal (e.g., cognitive processing) or vascular (e.g., hypercarbic $\mathrm{CO}_{2}$-induced) stimulation, cerebral blood flow (CBF) increases by much more than the cerebral metabolic rate of oxygen consumption $\left(\mathrm{CMRO}_{2}\right)$, leading to increased oxygenated red blood cells (carrying $\mathrm{HbO}$ ) in capillaries and veins. As deoxy-haemoglobin (Hb) is paramagnetic (counteracts main magnetic field, $\mathrm{BO}$, of the scanner), the relative increase in oxyhaemoglobin ( $\mathrm{HbO}_{2}$ ) leads to a more homogenous field in and around capillaries and veins, leading to a lengthening of the magnetic resonance image (MRI) relaxation time $\mathrm{T}_{2}\left(^{*}\right)$ and therefore increased MRI water signal. This effect is reduced primarily by increases in $\mathrm{CMRO}_{2}$ and small increases in venous CBV (which are much smaller than arterial CBV changes). This leads to an overall positive water signal increase in healthy tissue. B: Mean hypercarbic BOLD reactivity maps in patients $(n=50)$ with varying degrees of ischaemic disease reveal relatively symmetric reactivity patterns in average. C: Example of a patient with Moyamoya disease before and after surgical revascularisation demonstrates abilities for BOLD to serially monitor changes in cerebrovascular reactivity and reserve capacity. Colour bar denotes z-score with stimulus.

following perturbation by additional radiofrequency (B1) and gradient (G) fields. Water protons in different environments have unique relaxation properties and therefore can exhibit exquisite contrast between different healthy tissue types and between healthy and diseased tissue. Importantly and unlike contrast derived from positron emission tomography (PET), single-photon emission computed tomography (SPECT) or computed tomography (CT), different contrasts in MRI can be generated simply by adjusting scan parameters (e.g., B1 and G). This accelerates innovation and clinical implementation in MRI, as testing or approval that may be required from new exogenous tracers is irrelevant. However, a valid concern with MRI is that our ability to validate new techniques may be outpaced by diverging technical innovations. When this occurs, the clinical feasibility of new MR approaches and their true prognostic potential may be unclear. Here, we present an overview of several cerebrovascular imaging methods that have tested clinical potential and which deserve interest for implementation into large-scale clinical trials.

\section{Blood Oxygenation Level-dependent Cerebrovascular Reactivity}

Blood oxygenation level-dependent (BOLD) MR neuroimaging with predominately $\mathrm{T}^{*}$-weighted contrast ${ }^{15,16}$ and less commonly $\mathrm{T2}$-weighted contrast, ${ }^{17}$ has been widely applied to map brain activity ${ }^{18-21}$ non-invasively, transforming the field of cognitive neuroscience. BOLD contrast arises owing to the mismatch in cerebral blood flow (CBF), cerebral blood volume (CBV) and cerebral metabolic rate of oxygen $\left(\mathrm{CMRO}_{2}\right)$ increases secondary to both vascular and neuronal stimulation in healthy parenchyma. ${ }^{22}$ Specifically, CBF increases by generally 20-100 \% (depending on task), whereas $\mathrm{CMRO}_{2}$ increases only marginally by $0-20 \%{ }^{23,24}$ This leads to an increase in oxyhaemoglobin $\left(\mathrm{HbO}_{2}\right)$ relative to deoxyhaemoglogin $(\mathrm{Hb})$ in capillaries and veins. As $\mathrm{HbO}_{2}$ is diamagnetic whereas $\mathrm{Hb}$ is paramagnetic, the relative reduction of fractional $\mathrm{Hb}$ in the vasculature will increase surrounding water $\mathrm{T} 2$ and $\mathrm{T} 2 *$, thereby increasing MR signal. Additional dephasing arises from CBV increases in vasculature containing $\mathrm{Hb}$, which will reduce the surrounding water signal. ${ }^{25,26}$

BOLD MR was initially developed to assess changes in blood flow as a surrogate for neuronal activity, allowing functional localisation. BOLD signals are indirect markers of neuronal or vascular activity that arise consequential to modulations in haemodynamics and metabolism, ${ }^{20,21,27-34}$ In healthy tissue, coupling between these parameters are frequently preserved and therefore it is possible to make inferences regarding both haemodynamics and metabolism from ensemble BOLD signal under certain assumptions. However, when imaging diseased tissue, uncoupling between flow and metabolism, together with autoregulation complicates interpretation of the BOLD signal.22,35

Recently, BOLD imaging has been utilised in studies of cerebrovascular disease in conjunction with primarily isometabolic hypercarbic gas stimulation. ${ }^{36,37}$ Here, hypercarbia (generally $5 \% \mathrm{CO}_{2}$ ) is administered either with a balance of atmospheric air or $\mathrm{O}_{2}{ }^{38}$ The hypercarbic stimulus elicits relaxation of arterial smooth muscle and correspondingly increases in CBF and CBV. Therefore, the hypercarbic-induced BOLD signal is a marker of cerebrovascular reactivity (CVR) and the degree of increases in BOLD signal can be used to infer how near vasculature is to reserve capacity. This method has been used clinically in a range of patients with cervical and intracranial steno-occlusive disease ${ }^{3-41}$ and BOLD-measured CVR has been shown to correlate with angiographic and clinical measures of impairment, ${ }^{40}$ inversely with cortical thickness ${ }^{42}$ and variably with prognosis and stroke risk. ${ }^{43,44}$ Figure 1 shows an example of 
how BOLD CVR can be used clinically to provide additional information regarding parenchyma response to surgical revascularisation.

The advantages of BOLD CVR measurements are (i) a large existing literature demonstrating clinical feasibility, (ii) the non-invasive nature of the MRI protocol, which does not require intravenous contrast and (iii) simplicity of the imaging sequence (most commonly a wholebrain long echo time single-shot echo planar imaging acquisition). The disadvantages are (i) susceptibility artefacts around regions of air/ tissue interface in lower frontal lobe, (ii) contraindications in patients with severe pulmonary disease and (iii) necessity to monitor basic physiological changes including heart rate, blood pressure, end-tidal $\mathrm{CO}_{2}\left(\mathrm{EtCO}_{2}\right)$ and preferably fraction-inspired $\mathrm{O}_{2}$. Hypercarbic BOLD has been implemented with good success using standard non-rebreathing gas masks and commercial gas mixtures, although more elaborate and controlled gas delivery equipment using end-tidal forcing and similar procedures are also utilised. ${ }^{45}$

\section{Cerebral Blood Flow-weighted Arterial Spin Labelling}

Though arterial spin labelling (ASL) MRI is nearly as old as BOLD MRI, ${ }^{46}$ it is less widely used owing to greater technical hurdles related to acquisition. Contrary to BOLD, which provides a qualitative indirect marker for brain neurovascular function, ASL is primarily CBF-weighted, and with appropriate care can be used to quantify CBF in absolute units (millilitres blood/100 g tissue/minute), analogous to PET or CT perfusion imaging. In ASL, arterial blood water magnetisation is altered using either a single or more recently a string of radiofrequency pulses; this inverts the magnetisation of inflowing blood water relative to static tissue water. This inversion acts as an endogenous bolus. Blood water labelled in carotid and vertebra-basilar arteries travels through the large vasculature, enters the capillaries and exchanges with tissue water. Tissue signal is imaged after an adequate post-labelling delay time (inversion time, $\mathrm{TI}$ ) for this capillary exchange. The labelled blood water attenuates the tissue water signal very slightly (less than $1 \%$ of the static tissue signal) and therefore ASL generally requires a separate reference image to be acquired in which the blood water is not labelled. The difference between the label and reference image produces a CBF-weighted image and absolute quantification of CBF is possible upon application of the solution to the flow-modified Bloch equation for one (blood) or two (blood and tissue) compartments. ${ }^{47}$

Several variants of the original ASL approach have been implemented First, labelling can be performed using special radiofrequency transmit coils and a single, long pulse for highest SNR in the continuous ASL (CASL) method, or ASL can be performed using pulsed (PASL) or pseudo-continuous (PCASL) methods whereby a single or string of pulses are applied for labelling. Both PASL and PCASL can be performed using standard body coils for radiofrequency transmission, with PCASL providing nearly the same SNR as CASL. ${ }^{48}$ For these reasons, PCASL has recently emerged as the most popular ASL variant. Second, ASL applied at multiple post-labelling delays allows for simultaneous quantification of both CBF and arterial arrival time. When this method is repeated in the presence of bipolar gradients to dephase intravascular blood water signal, or alternatively with modelling procedures, it is additionally possible to quantify arterial $\mathrm{CBV}^{49-51}$ Finally, one can label blood water in different feeding arteries using vessel-encoded ASL to visualise perfusion territories. ${ }^{52}$ Figure 2 outlines the ASL principles and shows an example of ASL in patients with ischaemic cerebrovascular disease. Reviews of ASL application studies ${ }^{53}$ and validation with

\section{Figure 2: Arterial Spin Labelling Magnetic Resonance Imaging for Measuring Cerebral Blood Flow}

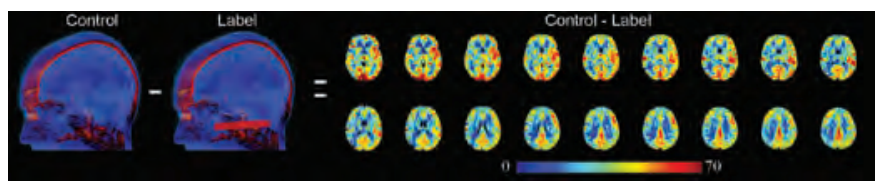

A: In arterial spin labelling (ASL), blood water is magnetically labelled through inversion of the arterial blood water magnetisation. The labelled bolus flows into the imaging slice and exchanges with tissue water, thereby slightly reducing the tissue water signal. This reduction in tissue water signal is better visualised when compared with a separate image in which the inflowing blood water is not labelled (e.g., 'control') and the difference between the label and control image provides a cerebral blood flow (CBF)-weighted map. The CBF can be quantified in absolute units upon application of the flow-modified Bloch equation or tracer kinetic models. Here, the CBF maps correspond to average maps from eight patients with asymmetric right-sided intracranial stenosis.

\section{Figure 3: Chemical Exchange Saturation Transfer Amide Proton Transfer Magnetic Resonance Imaging for Measuring $\mathrm{pH}$ and Macromolecule Content}

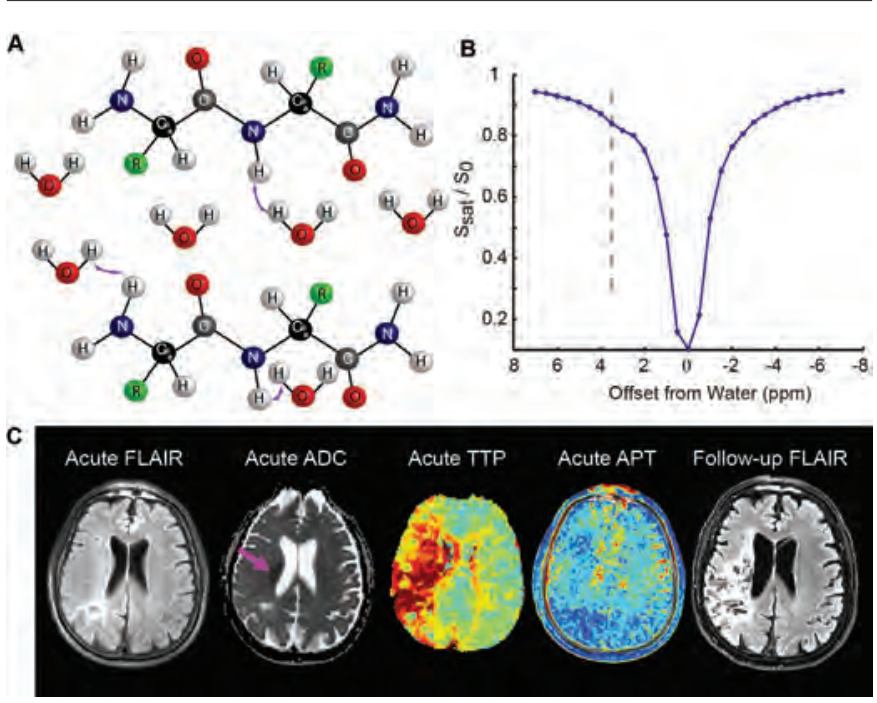

A: Amide protons $(\mathrm{N}-\mathrm{H})$ resonate at a unique frequency $3.5 \mathrm{ppm}$ from water protons. Therefore, prior to detection of the water signal, if an off-resonant radiofrequency pulse is applied at the amide resonance, the amide magnetisation will be altered. On the timescale of the magnetic resonance imaging (MRI) measurement the amide protons will exchange with water protons, which are detectable with standard imaging readouts. The signal reduction that is observed in the water signal following amide proton saturation and exchange varies with the amount of the amide protons in the voxel and the exchange rate, which is base-catalysed over a physiological range. B: In practice, radiofrequency irradiation is applied at different offsets relative to water Attenuation is observed at all offsets owing to multiple exchanging species, imperfect pulse performance and so-called 'spillover' effects; however, additional attenuation is observed at the amide proton transfer (APT) resonance $(3.5 \mathrm{ppm})$. By measuring the magnitude of this attenuation in different tissue types, it is possible to obtain information regarding exchange rate, $\mathrm{pH}$ and amide proton content. C: MRI performed 2-4 hours after acute onset of right middle cerebral artery stroke due to a middle cerebral artery occlusion in 58-year-old male: fluid attenuated inversion recovery (FLAIR) demonstrates encephalomalacia from remote stroke with mild hyperintense signal from oedema in the acute stroke in the centrum semiovale. The infarct core is identified on the apparent diffusion co-efficient (ADC) map by restricted diffusion (pink arrow) and is surrounded by a large penumbra, identified by delayed time to peak (TTP). Extensive decreased APT effect predicts final infarct volume on a follow-up MRI after 30 days more accurately than the ADC map, in this patient.

invasive techniques ${ }^{37}$ have recently been presented and principles of spin labelling have been extended to monitor $\mathrm{CSF}^{54}$ and lymphatic flow. ${ }^{55}$

The advantages of ASL are that it is safe (not requiring intravenous contrast) and quantitative, allowing longitudinal comparison of CBF during treatment. Furthermore, when performed in tandem with BOLD MRI, it possible to enhance quantitative interpretability of functional responses 
Figure 4: Ultra-high Field Relevance

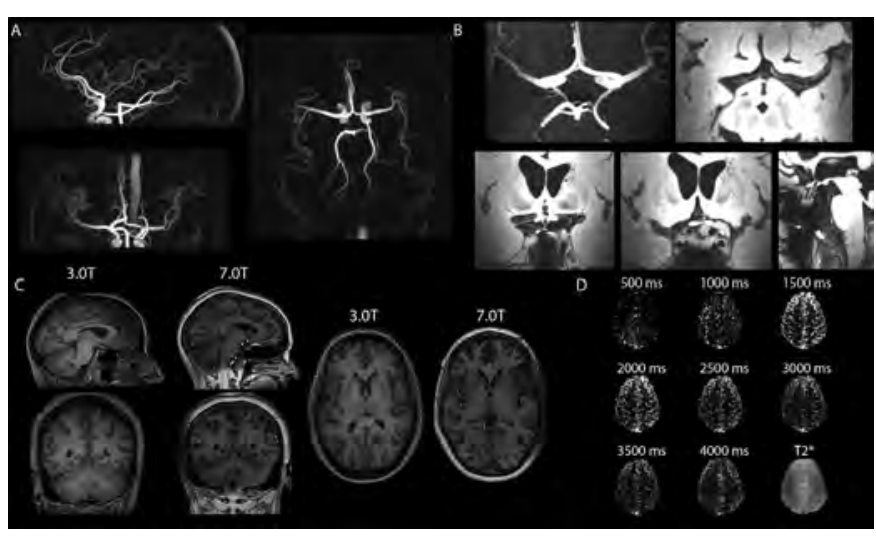

A: An example of $0.5 \mathrm{~mm}$ phase contrast angiography acquired at $7.0 \mathrm{~T}$ in approximately $4 \mathrm{~mm}$, along with $(B)$ arterial vessel wall imaging $(0.8 \mathrm{~mm})$ acquired in about 10 minutes. The vessel wall image shows clear vessel wall thickening in anterior cerebral artery and basilar artery. C: Standard T1-weighted imaging acquired at $3.0 T(1 \mathrm{~mm})$ and $7.0 T(0.7$ $\mathrm{mm}$ ) in the same volunteer in near-identical scan times of approximately 5 minutes. $D$ : Arterial spin labelling (ASL) acquired at different post-labelling delays demonstrates potential for measuring cerebral blood flow (CBF) at very long arterial arrival times (3-4 s), which are common in stroke. This phenomena is possible owing to the longer blood water T1 at 7.0T (2-2.5 s) relative to 3.0T (1.4-1.7 seconds).

by independently measuring CBF contributions to BOLD contrast, and, under appropriate modeling assumptions, infer changes in the remaining sources to BOLD contrast (e.g., CBV or $\mathrm{CMRO}_{2}$ ). Disadvantages of ASL are the low SNR (generally 10-20-fold lower than BOLD owing to the small effect of the blood water label) and reduced sensitivity to CBF in tissue with delayed arterial arrival times, including white matter or ischaemic tissue. The latter confound arises owing to the endogenous label decaying with the T1 of blood water, which is approximately $1.2 \mathrm{~s}$ at $1.5 \mathrm{~T}$ but grows to $1.6 \mathrm{~s}$ at $3.0 \mathrm{~T}^{56,57}$ Arterial arrival times are generally reported in the range of $0.7-1.1 \mathrm{~s}$, with an additional $1 \mathrm{~s}$ required for blood water to traverse the capillary bed. Therefore, ASL is more reliable at higher field strength where the blood water $\mathrm{T} 1$ (and in turn effective half-life of the tracer) is on the order of the blood-to-tissue transit time.

\section{pH-weighted Chemical Exchange Saturation Transfer}

While the MRI signal is almost always derived from protons on water molecules, it is also possible to indirectly image labile protons that exchange with water. One of the most popular methods for this is with amide proton transfer (APT) imaging. ${ }^{58,59}$ Whole-brain APT MRI can be obtained using standard equipment available at most hospitals and using commonplace imaging readouts optimised for structural and functional MRI. Chemical information is obtained through saturation transfer of magnetisation between amide protons (primarily on the peptide backbone; in vivo concentration $=70-100 \mathrm{mM}$ ) and the imaged water protons (concentration $=110 \mathrm{M}$ ). In APT, the labile protons originate from amide groups resonating at $8.3 \mathrm{ppm}$ in the proton spectrum (+3.5 ppm from water)..$^{60}$ Importantly, over a physiological $\mathrm{pH}(\mathrm{pH}>5)$, the exchange rate is base-catalysed and therefore the APT effect-size on the water signal reduces with decreasing $\mathrm{pH}$, or tissue acidosis. As anaerobic metabolism during ischaemia leads to considerable tissue acidosis, APT may permit insight into the energy status of ischaemic parenchyma by indirect pH imaging. Sun and colleagues have demonstrated potential for this method by monitoring the evolution of $\mathrm{pH}$ changes following middle cerebral artery occlusion in anaesthetised rats at 4.7T.61,62.

Variables predicting functional outcome following stroke include patient age, the National Institutes of Health Stroke Scale (NIHSS), ${ }^{63,64}$ the size of the infarct core ${ }^{65,66}$ and (more controversially) the presence of penumbra. ${ }^{67}$ Acute stroke intervention requires a judgment regarding the balance between revascularisation benefits and risks. Intravenous or intra-arterial thrombolysis and clot retrieval endovascular procedures have been shown to increase reperfusion rates and reduce final infarct size when administered within the first hours of symptom onset. ${ }^{68-70}$ However, reperfusing infarcted tissue increases the risk of haemorrhagic transformation, which occurs in approximately $6 \%$ of treated patients..$^{71}$ Much work has been performed to develop fast imaging tools that accurately identify both the ischaemic penumbra and the infarcted core of irreversibly damaged tissue. Clinically, this is most-often performed using perfusion CT or perfusion- and diffusion-weighted MRI (PWI and DWI). However, decreased perfusion may represent infarct core, penumbra and/or benign oligaemia.72-77 Imaging tissue acidosis may provide an additional method for characterising at risk and infarcted tissue in acute stroke. ${ }^{78}$ Figure 3 shows an example of an acute stroke patient imaged using pH-weighted APT CEST imaging.

The advantages of CEST imaging are that it is non-invasive and provides information regarding $\mathrm{pH}$ and macromolecular environment using typical imaging readouts already optimised from other modalities. This is in contrast to MR spectroscopy, which has been widely used for assessment of similar parameters, yet has extremely poor spatial coverage (generally one voxel) and is also time-intensive (5-15 minutes). A disadvantage of CEST imaging is that the nature of the contrast mechanism, including contributions from all exchanging species, has not been well summarised yet and therefore much development work is ongoing. Additionally, methodological CEST studies are frequently conducted at high field (>3.0T) where contrast is largest and it remains unclear how well this approach will perform at clinically available field strengths.

\section{Arterial Vessel Wall Imaging}

Cervical vascular stenosis is a well-known risk factor for stroke and much progress has been made in preventing strokes in this population. Following the North American Symptomatic Carotid Endarterectomy Trial (NASCET), patients with symptomatic high-grade cervical internal carotid artery (ICA) stenosis have been surgically revascularised with carotid endarterectomy, or more controversially, carotid stenting, ${ }^{79}$ reducing stroke incidence. Recently it has been shown that patients with IC stenosis are also at high risk for stroke, ${ }^{80}$ but the appropriate treatment for IC stenosis is less clear in the wake of the recently halted Stenting and Aggressive Medical Management for Preventing

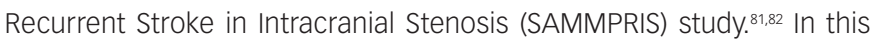
prospective trial, $14 \%$ of patients treated with angioplasty combined with stenting and medical management experienced a stroke or died within the first 30 days following treatment compared with $5.8 \%$ of patients who received medical management alone.

Arterial wall imaging of intracranial vessels is important for defining vulnerable plaque features, which have not been characterised for intracranial atherosclerotic disease and for improving management decisions. Much more is known about coronary and carotid bifurcation risk factors. For instance, nearly $70 \%$ of acute coronary events are caused by lesions that are not haemodynamically significant or flow limiting prior to the event. ${ }^{83}$ Carotid bifurcation studies have reported morphological plaque features are more predictive of stroke risk than luminal stenosis. ${ }^{84}$ Vulnerable extracranial plaque features, which have been validated in prospective studies and correlate with histopathological findings following carotid endarterectomy, include lipid-rich necrotic cores, intraplaque haemorrhage and torn fibrous caps. Improved understanding of the longitudinal progression of atherosclerosis has shown that early 
plaque is accommodated by external remodelling without luminal compromise, presumably in an attempt to preserve haemodynamic flow. Studies have demonstrated plaque burden and composition is a dynamic process, which can increase or decrease with time. ${ }^{85}$ Results from the recently completed Trial of Cilostazol in Symptomatic intracranial Stenosis 2 (TOSS-2) suggest MR may be sensitive to plaque changes in response to treatment. ${ }^{86}$ Preliminary findings of vulnerable intracranial plaque features on MRI include eccentric wall enhancement and intraplaque heterogeneity, which appears to correlate with embolic infarct patterns and ipsilateral stroke.

Once vulnerable plaque features are validated, the effects of current treatments, including anti-platelet therapy and statins, on plaque morphology could be monitored longitudinally with arterial wall imaging, which has recently been developed and studied in healthy volunteers and patients with cerebrovascular disease, utilising ultra-high spatial resolution methods. ${ }^{67-70}$ The general strategy in these methods is to null intravascular signal and CSF signal, revealing contrast between the vessel wall and the surrounding nulled regions. ${ }^{67}$ This can be achieved using a long turbo spin echo readout that will poorly refocus flowing spins through the region of interest, together with an inversion pre-pulse to null the Iongitudinal component of the blood water magnetisation. Other methods with similar goals exploit pulse trains in combination with a train of spoiler gradients. ${ }^{87}$ Figure 4 shows an example of vessel wall imaging in a patient with intracranial stenosis. The disadvantage of vessel wall imaging without contrast agents is that such protocols require sub-millimetre imaging of $0.5-0.8 \mathrm{~mm}$ to resolve vessel walls. Due to inherently low SNR at clinically available field strengths, this can necessitate 7-12 minutes of scan time. While feasible at a clinical field strength of 3.0T, the improved non-invasive vessel wall contrast has been reported at ultra-high field.

\section{Ultra-high Field Relevance}

Clinical magnetic field strengths are now common at both 1.5T and 3.0T, and approximately 50 research centres worldwide have 7.0T scanners. SNR increases linearly with field strength ${ }^{88}$ and thus identical spatial resolution is available in approximately half the scan time if the identical scan is performed at 3.0T compared with 1.5T. While SNR improvements are the most common impetus for moving to higher field (see Figure 4), additional beneficial changes in contrast occur as well. For instance, BOLD MRI contrast is extremely sensitive to draining veins, which carry elevated $\mathrm{HbO}_{2}$ during periods of neuronal and vascular stimulus. These cortical veins cause large susceptibility changes and therefore measurable increases in the T2(*)-weighted signal, which is not derived from vascular reactivity and may not co-localise with the functional area of interest. However, as magnetic field strength increases, the intravascular blood water $\mathrm{T} 2\left(^{*}\right)$ reduces relative to the extravascular tissue water $\left.\mathrm{T} 2{ }^{*}\right)$, which should reduce artifactual contamination from draining veins when BOLD is performed at higher field. At 1.5T, 3.0T and 7.0T the fraction of the BOLD effect that is extravascular is $0.54,0.78$, and 0.92 , respectively. ${ }^{99}$

Unlike T2(*), blood water T1 will increase with increasing magnetic field strength. ${ }^{57}$ This phenomena is particularly important for ASL, as the duration of the blood water label is proportional to the T1. Mean blood water (arterial and venous) T1 is approximately $1.2 \mathrm{~s}, 1.6 \mathrm{~s}$ and $2.3 \mathrm{~s}$ at 1.5T, 3.0T and 7.0T. Therefore, at 3.0T or higher ASL has increased potential for measuring CBF and arterial arrival times in tissue with reduced velocity and delayed arrival times, as is frequently the case in patients with vascular occlusion or severe stenosis (see Figure 4).

High field is particularly well-suited for APT imaging as water relaxation time lengthening, increased SNR and spectral dispersion at high field facilitates increased sensitivity to the chemical exchange effect at the time of imaging. There are very few drawbacks to performing clinical MRI at 3.0T relative to 1.5T, with the exception of implanted medical devices that may be contraindicated at higher field. However, this limitation is becoming less burdensome as more devices have been tested and approved at 3.0T. At 7.0T, very few medical devices have been tested and at many research centres, medical implanted devices of any kind are contraindicated. This substantially reduces the clinical relevance of 7.0T in the short term. Related to this concern, the specific absorption rate (SAR), defined as the RF power absorbed per unit mass, increases with increasing B0. This occurrence leads to increased heating and related safety concerns at high field, imposing limitations on pulse sequences that require short TR and high RF duty cycle (e.g., PCASL or pulse train CEST). More efforts are underway to evaluate the safety of implanted devices at 7.0T and the range of acceptable SAR constraints, however aside from promising reports of 7.0T feasibility, utilising 7.0T as a meaningful clinical tool has not yet been achieved in most centres.

\section{Conclusions and Summary}

Multiple non-invasive MRI approaches for evaluating tissue- and vascular haemodynamic impairment have been tested clinically. These include BOLD MRI for cerebrovascular reactivity evaluation, ASL for CBF quantification, CEST for PH-weighted contrast and AWI for vessel wall and plaque characterisation. The most advanced of these approaches in terms of clinical implementation are BOLD CVR and ASL CBF, with primarily feasibility testing having been performed in the remaining approaches. Finally, as all methods benefit greatly with increased magnetic field, 3.0T is essentially required and 7.0T even more promising once technical obstacles of ultra-high field imaging are addressed.
1. Donnan GA, Fisher, M, Macleod, M, et al., Stroke, Lancet, 2008;371:1612-23.

, Feigin VL, Stroke epide

Lancet, 2005;365:2160-

3. National Stroke Association, 2011 (Centennial, 2011).

4. Feigin V, Hoorn SV, How to study stroke incidence, Lancet, 2004;363:1920.

5. Langhorne P, Williams BO, Gilchrist W, et al., Do stroke units save lives?, Lancet, 1993;342:395-8,

6. Ng YS, Stein J, Ning M, et al., Comparison of clinical characteristics and functional outcomes of ischemic stroke in different vascular territories, Stroke, 2007;38:2309-14. Dobkin BH, Strategies for stroke rehabilitation, Lancet Neurol 2004;3:528-36.

8. Lai SM, Studenski S, Duncan PW, et al., Persisting consequences of stroke measured by the Stroke Impact Scale, Stroke, 2002;33:1840-4.

9. Duncan PW, Lai SM, Keighley J, Defining post-stroke recovery: implications for design and interpretation of drug trials, implications for design and interpretation

10. Lu H, Nagae-Poetscher LM, Golay X, et al., Routine clinical brain MRI sequences for use at 3.0 Tesla, J Magn Reson Imaging, 2005;22:13-22.
11. Noguchi K, Ogawa T, Inugami A, et al., Acute subarachnoid hemorrhage: MR imaging with fluid-attenuated inversion recovery pulse sequences, Radiology, 1995:196:773-7.

12. Deichmann R, Good CD, Josephs $\mathrm{O}$, et al., Optimization of 3-D MP-RAGE sequences for structural brain imaging Neuroimage, 2000;12:112-27.

13. Jones JG, Cen SY, Lebel RM, et al., Diffusion tensor imaging correlates with the clinical assessment of disease severity in cervical spondylotic myelopathy and predicts outcome following surgery, AJNR Am J Neuroradiol, 2013;34:471-8.

14. Van Zijl PC, Barker, PB, Magnetic resonance spectroscopy and spectroscopic imaging for the study of brain metabolism, Ann N Y Acad Sci, 1997;820:75-96.

15. Ogawa S, Lee TM, Kay AR, et al., Brain magnetic resonance imaging with contrast dependent on blood oxygenation, Proc Natl Acad Sci U S A, 1990;87:9868-72.

16. Kwong KK, Belliveau JW, Chesler DA, et al., Dynamic magnetic resonance imaging of human brain activity during primary resonance imaging of human brain activity during primary

17. Yacoub E, Duong TQ, Van De Moortele PF, et al., Spin-echo fMRI in humans using high spatial resolutions and high magnetic fields, Magn Reson Med, 2003;49:655-64.

18. Ogawa S, Lee TM, Kay AR, et al., Brain magnetic resonance imaging with contrast dependent on blood oxygenation, Proc Natl Acad Sci U S A, 1990:87:9868-72.

19. Kim DS, Ronen I, OIman C, et al., Spatial relationship between neuronal activity and BOLD functional MRI, Neuroimage, 2004;21:876-85

20. Fischer JE, Ramser M, Altermatt S, et al., [Rational utilization of antibiotics in critically ill children], Schweiz Med Wochenschr, 2000;130:1564-71

21. van Zijl PC, Eleff SM, Ulatowski JA, et al., Quantitative assessment of blood flow, blood volume and blood oxygenation effects in functional magnetic resonance imaging, Nat Med, 1998;4:159-67.

22. Blicher JU, Stagg CJ, O'Shea J, et al., Visualization of altered neurovascular coupling in chronic stroke patients using multimodal functional MRI I Cereb Blood Flow Metab, 2012;32:2044-54.

23. Donahue MJ, Blicher JU, Ostergaard L, et al., Cerebral blood flow, blood volume, and oxygen metabolism dynamics in human visual and motor cortex as measured by whole-brain multi-modal magnetic resonance imaging, I Cereb Blood Flow Metab, 2009;29:1856--66.

24. Lu H, Golay X, Pekar JJ, et al., Sustained poststimulus elevation in cerebral oxygen utilization after vascular recovery, J Cereb 
Blood Flow Metab, 2004:24:764-70

25. Moon CH, Fukuda M, Kim SG, Spatiotemporal characteristics and vascular sources of neural-specific and -nonspecific fMR signals at submillimeter columnar resolution, Neuroimage, 2013;64:91-103

26. van Zijl PC, Eleff SM, Ulatowski JA, et al., Quantitative assessment of blood flow, blood volume and blood oxygenation effects in functional magnetic resonance imaging, Nat Med, 1998;4:159-67.

27. Chappell MA, Maclntosh BJ, Donahue MJ, et al., Separation of macrovascular signal in multi-inversion time arterial spin labelling MRI, Magn Reson Med, 2010;63:1357-365.

28. Muthukumaraswamy SD, Edden RA, Jones DK, et al., Resting GABA concentration predicts peak gamma frequency and fMRI amplitude in response to visual stimulation in humans, Proc Natl Acad Sci U S A, 2009:106:8356-61.

29. Enager P, Piilgaard $\mathrm{H}$, Offenhauser $\mathrm{N}$, et al., Pathway-specific variations in neurovascular and neurometabolic coupling in rat primary somatosensory cortex, I Cereb Blood Flow Metab 2009;29:976-86

30. Donahue MJ, Blicher JU, Ostergaard L, et al., Cerebral blood flow, blood volume, and oxygen metabolism dynamics in human visual and motor cortex as measured by whole-brain multi-modal magnetic resonance imaging, I Cereb Blood Flow Metab, 2009;29:1856-66.

31. Qiu M, Ramani R, Swetye M, et al., Anesthetic effects on regional $\mathrm{CBF}, \mathrm{BOLD}$, and the coupling between task-induced changes in CBF and BOLD: an FMRI study in normal human subjects, Magn Reson Med, 2008;60:987-96.

32. Shulman RG, Hyder F, Rothman DL, Biophysical basis of brain activity: implications for neuroimaging, $Q$ Rev Biophys, 2002;35:287-325.

33. Selmer IS, Schindler M, Humphrey PP, et al., First localisation of somatostatin sst(4) receptor protein in selected human brain areas: an immunohistochemical study, Brain Res $\mathrm{MO}$ Brain Res, 2000;82:114-25.

34. Kim SG, Rostrup E, Larsson HB, et al., Determination of relative CMRO2 from CBF and BOLD changes: significant increase of oxygen consumption rate during visual stimulation, Magn Reson Med, 1999;41:1152-61.

35. Derdeyn $\mathrm{CP}$, Videen $\mathrm{TO}$, Yundt $\mathrm{KD}$, et al., Variability of cerebral blood volume and oxygen extraction: stages of cerebral haemodynamic impairment revisited, Brain 2002;125:595-607.

36. Mikulis, DJ, Functional cerebrovascular imaging in brain ischemia: permeability, reactivity, and functional MR imaging, Neuroimaging Clin N Am, 2005;15:667-80, xii.

37. Donahue MJ, Strother MK, Hendrikse J, Novel MRI approaches for assessing cerebral hemodynamics in ischemic cerebrovascular disease, Stroke, 2012;43:903-15.

8. Wise RG, Harris AD, Stone AJ, et al., Measurement of OEF and absolute CMRO: MRI-based methods using interleaved and combined hypercapnia and hyperoxia, Neuroimage, 2013;83C:135-47.

39. Mikulis DJ, Krolczyk G, Desal H, et al., Preoperative and postoperative mapping of cerebrovascular reactivity in moyamoya disease by using blood oxygen level-dependent magnetic resonance imaging, J Neurosurg, 2005:103:347-55.

40. Donahue MJ, Ayad M, Moore R, et al., Relationships between hypercarbic reactivity, cerebral blood flow, and arterial circulation times in patients with moyamoya disease, J Magn Reson Imaging, 2013;38:1129-39.

41. Mandell DM, Han JS, Poublanc J, et al., Mapping cerebrovascular reactivity using blood oxygen leveldependent MRI in Patients with arterial steno-occlusive disease: comparison with arterial spin labeling MRI Stroke, 2008;39:2021-8.

42. Fierstra J, Poublanc J, Han JS, et al., Steal physiology is spatially associated with cortical thinning, J Neurol Neurosurg Psychiatry, 2010;81:290-3.

43. Conklin J, Fierstra J, Crawley AP, et al., Mapping white matter diffusion and cerebrovascular reactivity in carotid occlusive disease, Neurology, 2011;77:431-8.

44. Han JS, Abou-Hamden A, Mandell DM, et al., Impact of extracranial-intracranial bypass on cerebrovascular reactivity and clinical outcome in patients with symptomatic moyamoya vasculopathy, Stroke, 2011:42:3047-54.

45. Wise RG, Pattinson KT, Bulte DP, et al., Dynamic forcing of end-tidal carbon dioxide and oxygen applied to functional magnetic resonance imaging, I Cereb Blood Flow Metab 2007;27:1521-32.

46. Williams DS, Detre JA, Leigh JS, et al., Magnetic resonance imaging of perfusion using spin inversion of arterial water, Proc Natl Acad Sci U S A, 1992;89:212-6.

47. Zhou J, Wilson DA, Ulatowski JA, et al., Two-compartment exchange model for perfusion quantification using arterial spin tagging, I Cereb Blood Flow Metab, 2001;21:440-55.

48. Wu WC, Fernandez-Seara M, Detre JA, et al., A theoretical and experimental investigation of the tagging efficiency of pseudocontinuous arterial spin labeling, Magn Reson Med, 2007;58:1020-7.

49. MacIntosh BJ, Filippini N, Chappell MA, et al., Assessmen of arterial arrival times derived from multiple inversion me pulsed arterial spin labeling MRI, Magn Reson Med, 2010;63:641-7.

50. Chappell MA, MacIntosh BJ, Donahue MJ, et al., Separation of macrovascular signal in multi-inversion time arterial spin labelling MRI, Magn Reson Med, 2010;63:1357-65

51. Petersen ET, Lim T, Golay X, Model-free arterial spin labeling quantification approach for perfusion MRI, Magn Reson Med 2006;55:219-32.

52. Wong EC, Vessel-encoded arterial spin-labeling using pseudocontinuous tagging, Magn Reson Med, 2007;58:1086-91

53. Detre JA, Rao H, Wang DJ, et al., Applications of arterial spin labeled MRI in the brain, I Magn Reson Imaging, 2012;35:1026-37.

54. Yamada S, Miyazaki M, Kanazawa H, et al., Visualization of cerebrospinal fluid movement with spin labeling at MR imaging: preliminary results in normal and pathophysiologic Conditions, Radiology, 2008:249:644-52.

55. Rane S, Donahue PM, Towse T, et al., Clinical feasibility of noninvasive visualization of lymphatic flow with principles of spin labeling mr imaging: implications for lymphedema assessment, Radiology, 2013;269(3):893-902.

56. Lu H, Clingman C, Golay X, et al., Determining the Iongitudina relaxation time (T1) of blood at 3.0 Tesla, Magn Reson Med 2004;52:679-82.

57. Rooney WD, Johnson G, Li X, et al., Magnetic field and tissue dependencies of human brain longitudinal $1 \mathrm{H} 2 \mathrm{O}$ relaxation in vivo, Magn Reson Med, 2007; 57:308-18.

58. Zhou J, Payen JF, Wilson DA, et al., Using the amide proton signals of intracellular proteins and peptides to detect $\mathrm{pH}$ effects in MRI, Nat Med, 2003:9:1085-90.

59. Ward KM, Aletras AH, Balaban RS, A new class of contrast agents for MRI based on proton chemical exchange dependent saturation transfer (CEST), J Magn Reson 2000;143:79-87.

60. van Zijl PC, Zhou J, Mori N, et al., Mechanism of magnetization transfer during on-resonance water saturation. A new approach to detect mobile proteins, peptides, and lipids, Magn Reson Med, 2003;49:440-9.

61. Sun PZ, Zhou J, Sun W, et al., Detection of the ischemic penumbra using pH-weighted MRI, I Cereb Blood Flow Metab, 2007;27:1129-36.

62. Sun PZ, Cheung JS, Wang E, et al., Association between $\mathrm{pH}$-weighted endogenous amide proton chemical exchange saturation transfer MRI and tissue lactic acidosis during acute ischemic stroke, J Cereb Blood Flow Metab, 2011;31:1743-50.

63. Nakayama H, Jorgensen HS, Raaschou HO, et al., The influence of age on stroke outcome. The Copenhagen Stroke Study, Stroke 1994:25:808-13.

64. Adams HP, Jr., Davis PH, Leira EC, et al., Baseline NIH Stroke Scale score strongly predicts outcome after stroke: A report of the Trial of Org 10172 in Acute Stroke Treatment (TOAST), Neurology, 1999;53:126-31

65. Jovin TG, Yonas H, Gebel JM, et al., The cortical ischemic core and not the consistently present penumbra is a determinant of clinical outcome in acute middle cerebral artery occlusion, Stroke, 2003;34:2426-33.

66. Gasparotti R, Grassi M, Mardighian D, et al., Perfusion CT in patients with acute ischemic stroke treated with intra-arteria thrombolysis: predictive value of infarct core size on clinical outcome, AJNR Am J Neuroradiol, 2009;30:722-7.

67. Zhu G, Michel P, Aghaebrahim A, et al., Prediction of recanalization trumps prediction of tissue fate: the penumbra: a dual-edged sword, Stroke, 2013:44:1014-9.

68. Hacke W, Kaste M, Bluhmki E, et al., Thrombolysis with alteplase 3 to 4.5 hours after acute ischemic stroke, N Eng/ J Med, 2008;359:1317-29.

69. Wechsler $L R$, Intravenous thrombolytic therapy for acut ischemic stroke, N Eng/ J Med, 2011;364:2138-46.

70. Hesselmann V, Niederstadt T, Dziewas R, et al., Reperfusion by combined thrombolysis and mechanical thrombectomy in acute stroke: effect of collateralization, mismatch, and time to and grade of recanalization on clinical and tissue outcome AJNR Am J Neuroradiol, 2012;33:336-42.

71. Broderick JP, Palesch YY, Demchuk AM, et al., Endovascular therapy after intravenous t-PA versus t-PA alone for stroke, N Eng/J Med, 2013;368:893-903.

72. Fiehler I, Foth M, Kucinski T, et al, Severe ADC decreases do not predict irreversible tissue damage in humans, Stroke, 2002;33:79-86

73. Rivers CS, Wardlaw JM, What has diffusion imaging in animals told us about diffusion imaging in patients with ischaemic stroke?, Cerebrovasc Dis, 2005;19:328-36.

74. Parsons MW, Yang Q, Barber PA, et al., Perfusion magnetic resonance imaging maps in hyperacute stroke: relative cerebral blood flow most accurately identifies tissue destine to infarct, Stroke, 2001;32:1581-7.

75. Kamalian S, Konstas AA, Maas MB, et al., CT perfusion mean transit time maps optimally distinguish benign oligemia from true 'at-risk' ischemic penumbra, but thresholds vary by postprocessing technique, AJNR Am I Neuroradiol 2012;33:545-9.

76. Kidwell CS, Alger JR, Saver JL, Evolving paradigms in neuroimaging of the ischemic penumbra, Stroke,
$2004 \cdot 35 \cdot 2662-5$

Rivers CS, Wardlaw JM, Armitage PA, et al., Do acute diffusionand perfusion-weighted MRI lesions identify final infarc volume in ischemic stroke?, Stroke, 2006;37:98-104.

78. Hossmann KA, Cerebral ischemia: models, methods and outcomes, Neuropharmacology, 2008;55:257-70.

9. Clinical alert: benefit of carotid endarterectomy for patients with high-grade stenosis of the internal carotid artery. National Institute of Neurological Disorders and Stroke Stroke and Trauma Division. North American Symptomatic Carotid Endarterectomy Trial (NASCET) investigators, Stroke, 1991;22:816-7.

80. Ovbiagele B, Cruz-Flores S, Lynn MJ, et al., Early stroke risk after transient ischemic attack among individuals with after transient ischemic attack among individuals with 2008;65:733-7.

81. Qureshi Al, Interpretation and implications of the prematurely terminated Stenting and Aggressive Medical Management for Preventing Recurrent Stroke in the Intracranial Stenosis (SAMMPRIS) trial, Neurosurgery, 2012;70:E264-8.

82. Marks MP, Is there a future for endovascular treatment of intracranial atherosclerotic disease after Stenting and Aggressive Medical Management for Preventing Recurrent Stroke and Intracranial Stenosis (SAMMPRIS)?, Stroke, 2012;43:580-4.

83. Falk E, Shah PK, Fuster V, Coronary plaque disruption, Circulation, 1995;92:657-71.

84. van Gils MJ, Vukadinovic D, van Dijk AC, et al., Carotid atherosclerotic plaque progression and change in plaque composition over time: a 5 -year follow-up study using seria CT angiography, AJNR Am I Neuroradiol, 2012;33:1267-73.

85. Underhill HR, Yuan C, Zhao XQ et al., Effect of rosuvastatin therapy on carotid plaque morphology and composition in moderately hypercholesterolemic patients: a high-resolution magnetic resonance imaging trial, Am Heart $\mathrm{J}$ 2008; 155:584 e581-8.

86. Kim, DE, Kim, JY, Jeong, SW, et al., Association between changes in lipid profiles and progression of symptomatic intracranial atherosclerotic stenosis: a prospective multicenter study, Stroke, 2012;43:1824-30.

87. Li L, Miller KL, Jezzard P, DANTE-prepared pulse trains: a novel approach to motion-sensitized and motion-suppressed quantitative magnetic resonance imaging, Magn Reson Med, 2012;68:1423-38.

88. Triantafyllou C, Polimeni JR, Wald LL, Physiological noise and signal-to-noise ratio in fMRI with multi-channel array coils, Neuroimage, 2011;55:597-606.

89. Donahue MJ, Hoogduin $\mathrm{H}$, van Zijl PC, et al., Blood oxygenation level-dependent (BOLD) total and extravascular signal changes and DeltaR2* in human visual cortex at 1.5, 3.0 and 7.0 T, NMR Biomed, 2011;24:25-34.

90. Mandell DM, Han JS, Poublanc J, et al., Quantitative measurement of cerebrovascular reactivity by blood oxyge level-dependent MR imaging in patients with intracranial stenosis: preoperative cerebrovascular reactivity predicts the effect of extracranial-intracranial bypass surgery, AJNR Am I Neuroradiol, 2011;32:721-7.

91. Buxton RB, Wong EC, Frank LR, Dynamics of blood flow and oxygenation changes during brain activation: the balloon model, Magn Reson Med, 1998;39:855-64

92. Tancredi FB, Gauthier CJ, Madjar C, et al., Comparison of pulsed and pseudocontinuous arterial spin-labeling for measuring $\mathrm{CO} 2$-induced cerebrovascular reactivity, I Magn Reson Imaging, 2012:36:312-21.

93. Chalela JA, Alsop DC, Gonzalez-Atavales JB, et al., Magnetic resonance perfusion imaging in acute ischemic stroke usin continuous arterial spin labeling, Stroke, 2000;31:680-7.

94. Detre JA, Alsop DC, Perfusion magnetic resonance imaging with continuous arterial spin labeling: methods and clinical applications in the central nervous system, Eur J Radiol, 1999;30:115-24.

95. Okell TW, Chappell MA, Kelly ME, et al., Cerebral blood flow quantification using vessel-encoded arterial spin labeling j Cereb Blood Flow Metab, 2013;33:1716-24.

96. van der Kolk $A G$, Zwanenburg JJ, Brundel $\mathrm{M}$, et al., Intracrania vessel wall imaging at 7.0-T MRI, Stroke, 2011;42:2478-84.

97 Mandell DM, Matouk CC, Farb RI, et al., Vessel wall MRI to differentiate between reversible cerebral vasoconstriction syndrome and central nervous system vasculitis: preliminary results, Stroke, 2012:43:860-2.

98. van der Kolk AG, Hendrikse J, Brundel M, et al., Multisequence whole-brain intracranial vessel wall imaging at 7.0 tesla, Eur Radiol, 2013;23:2996-3004

99. Sun PZ, Zhou J, Sun W, et al., Detection of the ischemic penumbra using pH-weighted MRI, I Cereb Blood Flow Metab, 2007;27:1129-36.

100. Tietze A, Blicher J, Mikkelsen IK, et al., Assessment of ischemic penumbra in patients with hyperacute stroke using amide proton transfer (APT) chemical exchange saturation transfer (CEST) MRI, NMR Biomed, 2013; Epub ahead of print.

101. van Zijl PC, Yadav NN, Chemical exchange saturation transfer (CEST): what is in a name and what isn't?, Magn Reson Med, 2011;65:927-48. 